\title{
LOS SESGOS COGNITIVOS: DE LA PSICOLOGÍA COGNITIVA A LA PERSPECTIVA COGNITIVA DE LA ORGANIZACIÓN Y SU RELACIÓN CON LOS PROCESOS DE TOMA DE DECISIONES GERENCIALES
}

\section{The cognitive biases: From cognitive psychology to the cognitive perspective of the organization, and its relationship with the processes to make management decisions}

\section{Lorena Barón}

Profesora-investigadora titular del Decanato de Ciencias Económicas y Empresariales de la Universidad Centroccidental Lisandro Alvarado, Venezuela. E-mail: lorenabaron@gmail.com.

Gerardo J. Zapata Rotundo

Profesor-investigador titular del Decanato de Ciencias Económicas y Empresariales de la Universidad Centroccidental Lisandro Alvarado, Venezuela. E-mail: zapager@yahoo.com.

Recibido: 25/8 2017 • Aprobado: 25/11/2017

\section{Resumen}

La presente investigación, de naturaleza teórico-descriptiva, tiene como objetivo estudiar y analizar los sesgos cognitivos bajo la perspectiva de la toma de decisiones gerenciales. El tema se aborda a partir de una revisión bibliográfica de los principales postulados de la psicología cognitiva, comenzando con los aportes de March y Simon, quienes son reconocidos como los autores seminales de esta perspectiva teórica, y continuando con diferentes autores que la han estudiado y aplicado dentro del marco de las organizaciones, formando así la escuela cognitiva de la organización. En el transcurso del trabajo se desarrolla la relación entre los sesgos cognitivos y el proceso de percepción gerencial, para luego ofrecer un conjunto de contribuciones de la literatura que muestra aspectos vinculados con su medición, sus implicaciones y sus aplicaciones. Producto de esta revisión, se destaca, a modo de conclusión, el gran potencial que tienen los procesos y los sesgos cognitivos para entender y explicar el comportamiento de la gerencia y los líderes de la organización frente a situaciones de alta complejidad, que tienen efectos muy importantes en su desempeño.

Palabras clave: desarrollo organizacional; psicología cognitiva; procesamiento cognitivo; sesgos cognitivos y toma de decisiones.

\begin{abstract}
The present research of a theoretical-descriptive nature aims to study cognitive biases in the organization from the perspective of management decision making. The subject is approached from a bibliographical review of the main postulates of cognitive psychology, and the beginnings of its applications in the organization according to the contributions of March and Simon, recognized as seminal authors for the development of this theoretical perspective, and continuing with different authors who have adopted and addressed it within the framework of the study of organizations, thus forming the cognitive school of the organization. During the work, the relationship between cognitive biases and the managerial perception process is developed, to then offer a set of contributions from the literature that show aspects related to their measurement and implications and applications. Because of this review, we highlight the great potential of processes and cognitive biases to understand and explain the behavior of management and leaders of the organization in situations of high complexity that have very important effects on their performance.
\end{abstract}

Keywords: organizational development; cognitive psychology; cognitive processing; cognitive biases; and decision making. 


\section{Introducción}

La teoría cognitiva de la organización forma parte del objeto de estudio del llamado management estratégico; este abarca los distintos enfoques de pensamiento desarrollados dentro del marco de las organizaciones, que contribuyen con la definición de sus estrategias, las cuales incluyen su diseño, planificación, formulación, implementación y control (Mintzberg, Ahlstrand \& Lampel, 1999), formando así la escuela cognitiva de la organización. Sus fundamentos se recogen en una serie de investigaciones cuyo enfoque se orienta a estudiar la mente del directivo, específicamente en la formación de la estrategia, utilizando para ello los postulados de la psicología cognitiva.

Por su parte, Schwenk (1995) la describe como una perspectiva teórica con implicaciones prácticas muy importantes, orientada a los procesos que influyen en la toma de decisiones, desde la conceptualización del plan estratégico, la manera como se definen los marcos de implementación y actuación, hasta la definición de ciertas características de actuación y comportamiento de los directivos y de las organizaciones que dirigen. Ampliando este concepto, según Zapata \& Hernández (2014), la perspectiva cognitiva centra su atención en los procesos de toma de decisiones en condiciones complejas, dinámicas e inciertas, enfocados desde el punto de vista de la cognición del individuo.

La toma de decisiones es una actividad inherente a los procesos gerenciales, tanto desde el punto de vista del individuo como de la organización, entendida esta como un sistema integrado de individuos. Esto implica la consideración de aspectos complejos, tales como las condiciones del entorno cambiante y competitivo, la información disponible sobre las fortalezas y debilidades de la organización frente a ese entorno, las decisiones tomadas en el pasado y sus efectos en los resultados de la empresa y su proyección futura, las decisiones tomadas por otras organizaciones y sus consecuencias en las relaciones inter-organizacionales, las expectativas sobre los resultados, la cultura organizacional, entre otros.

Según Simon (1947), el ser humano percibe al mundo como un modelo simplificado de la gran confusión y complejidad del mundo real, y en su esfuerzo por alcanzar la racionalidad se ve restringido dentro de los límites de sus propios conocimientos. Esta idea constituye, en esencia, el punto de partida para definir el concepto de la racionalidad limitada, aspecto medular para la construcción y el desarrollo de la teoría cognitiva de la organización. De este modo, las ideas desarrolladas por Simon sobre la racionalidad limitada plantean que el hombre económico clásico toma decisiones óptimas en un ambiente claramente conocido y bien definido; al contrario del hombre administrativo, que solo puede considerar aspectos fraccionados de la realidad (March \& Simon, 1958). Una parte importante de esta visión fraccionada está determinada por los sesgos cognitivos, considerados como filtros o predisposiciones mentales particulares de cada individuo que condicionan su pensamiento $y$, en consecuencia, su actuación (Zapata \& Canet, 2009).

El objetivo central de este trabajo gira en torno al estudio de los sesgos cognitivos y su relación con los procesos de toma de decisiones gerenciales, partiendo de los conceptos de la psicología cognitiva y de la perspectiva cognitiva de la organización, de manera más específica. El tema se aborda a partir de una revisión bibliográfica de los principales postulados de la psicología cognitiva y de los inicios de sus aplicaciones en la organización, de acuerdo con los aportes de March y Simon, quienes son reconocidos como los autores seminales de la teoría cognitiva de la organización. Se establece la relación entre los sesgos cognitivos y el proceso cognitivo de la percepción de quienes dirigen la empresa, y se ofrecen posteriormente diferentes contribuciones de la literatura vinculadas con la medición de los sesgos cognitivos y con algunas de sus implicaciones en 
el comportamiento de la organización. Finalmente, se presenta un apartado con las principales conclusiones de la investigación.

\section{Fundamentación teórica}

\section{De la psicología cognitiva a la perspectiva cog- nitiva de la organización}

La psicología cognitiva surge como respuesta a lo que se conoce como psicología del sentido común o conductismo, apoyada en la idea de que las personas actúan basadas en el conocimiento o en las representaciones internas del significado del mundo, con criterios de objetividad. Aunque ambas corrientes coexisten, es la noción de objetividad la que ha sido duramente criticada, lo cual ha dado espacio al desarrollo del término de la complejidad mental (Riviere, 1991).

De este modo, la publicación del libro de Neisser, en 1967, titulado Cognitive Psychology, representa el símbolo del comienzo de una nueva época, como respuesta al modelo conductista que considera estudios de carácter científico solo aquellos que se centran en propiedades observables del comportamiento, analizadas con relación a los estímulos ambientales. Así, la psicología cognitiva centra su atención en procesos no observables que involucran funciones complejas del pensamiento, tales como: percepción, memoria, lenguaje, formación de conceptos y solución de problemas (Escobedo, 1993). Para Gardner (1987), la psicología cognitiva estudia temas relacionados con la mente, como la aceptación de los límites inherentes a la cantidad de información que los sujetos pueden recibir, los pasos para el procesamiento de esa información y la revisión de las estrategias globales empleadas para resolver problemas.

Las funciones mentales también han sido objeto de estudio de la filosofía y por esa razón se observan planteamientos de los grandes filósofos en los trabajos de los psicólogos cognitivos, con la diferencia de que estos últimos han tratado de comprobar experimentalmente las hipótesis a las que pretenden dar respuesta y, en función de los resultados, establecer modelos y conclusiones. Otra característica de la psicología cognitiva es la analogía que establece entre el pensamiento humano y el funcionamiento de un computador, utilizando términos propios de la informática para analizar y describir estos procesos (Escobedo, 1993).

En otro sentido, March \& Simon (1958) estudian las características del comportamiento humano en las organizaciones, partiendo de la premisa de considerar una organización como un organismo selector que debe tomar decisiones y resolver problemas, para lo cual está limitada en cuanto a la cantidad de opciones que puede atender al mismo tiempo; esto se debe a que la cantidad de información recogida y disponible en su memoria y la que está presente en el ambiente a su alcance es también limitada. Los autores describen el aprendizaje como un procedimiento lento, determinado por los cambios en el contenido total de la memoria.

Lo antes descrito forma parte de los intentos precursores de utilizar el campo de estudio de la psicología cognitiva en el management estratégico, en lo que hoy se conoce como la perspectiva cognitiva de la organización. Para autores como Mintzberg et al. (1999), esta perspectiva -más que una escuela cerrada de pensamiento- se considera como un cuerpo de investigaciones libres que puede llegar a transformar la enseñanza y la práctica de la estrategia desde el punto de vista de la cognición. Por su parte, Zapata \& Hernández (2014) plantean que tal perspectiva pretende analizar por qué los miembros de la organización actúan de determinada manera, cómo toman decisiones frente a distintas circunstancias $y$, finalmente, en qué consiste el proceso de decisión estratégica, esto según los conceptos y aspectos relacionados con la cognición del individuo.

Las implicaciones de un proceso de toma de decisiones pasan por la revisión de los procesos cognitivos. 


\section{Figura 1. Niveles de análisis del fenómeno cognitivo}

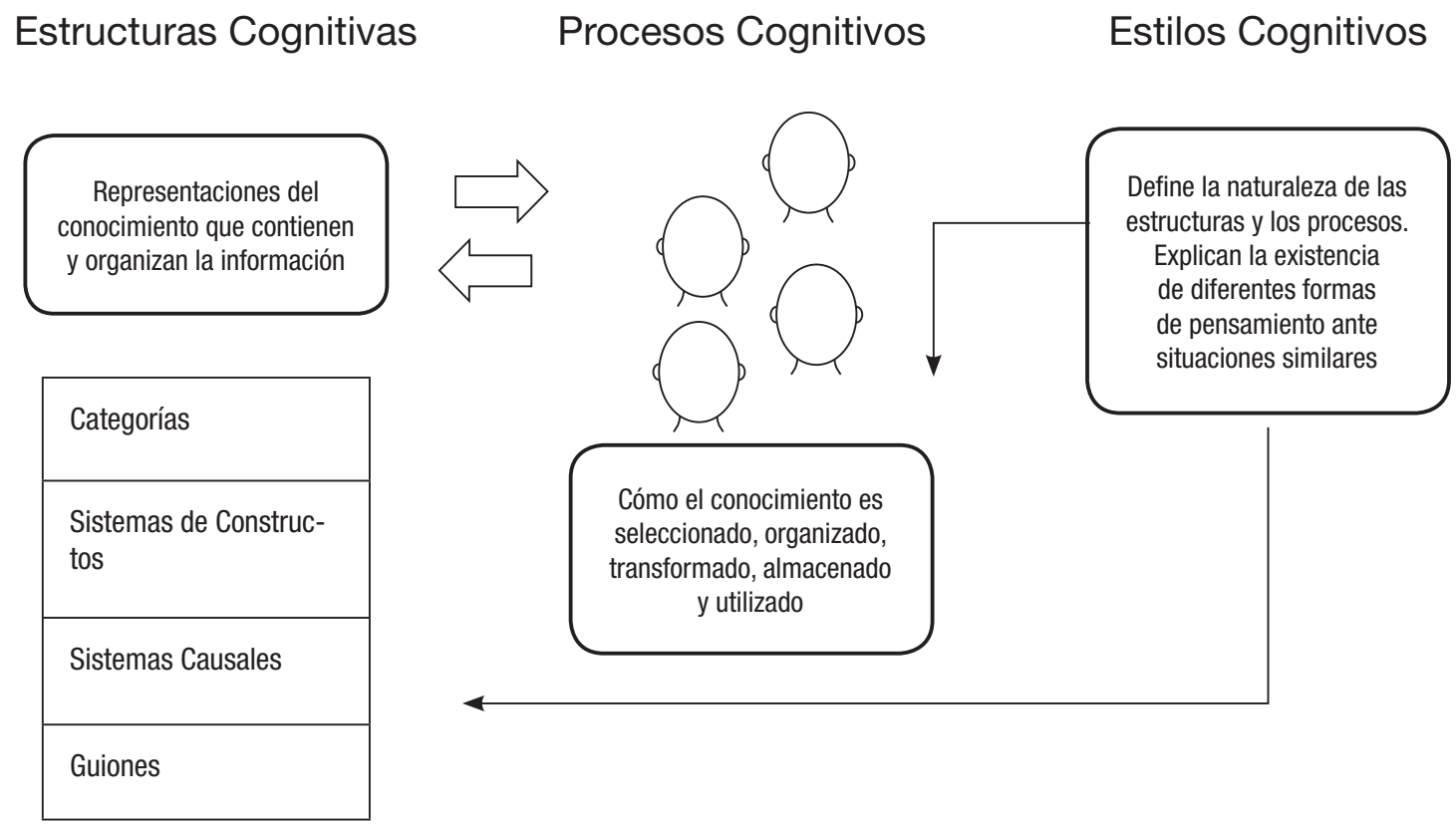

Fuente: Elaboración propia basada en el trabajo de Zapata \& Hernández (2014).

Para Banyard, Cassells, Green, Hartland, Hayes \& Reddy (1995), la cognición se relaciona con el pensamiento y la comprensión; es la expresión de cómo funciona la mente humana. Según estos autores, esta es el resultado de una serie de procesos: percepción, atención, pensamiento, memoria y lenguaje; es un proceso humano de carácter psicológico que tiene lugar a nivel individual, pero está fuertemente influido por sistemas sociales como, por ejemplo, las organizaciones.

Para autores como Nonaka (1994), las estructuras del conocimiento organizacional se construyen sobre la base del aprendizaje individual y la cognición de quienes la integran; esto quiere decir que el conocimiento individual se comparte y se institucionaliza para convertirse en el sistema de conocimiento de la organización. Esta idea es respaldada por Weick (1969), quien propone un enfoque psicológico de la organización expresado en función de las tareas que implica el proceso de organizar. Desde esta perspectiva, la organización y, por ende, la función de organizar es básicamente un proceso cognitivo que debe ser reconocido en sus diferentes niveles de análisis. Gráficamente, estos niveles podrían representarse como se indica en la figura 1.

En la figura anterior se distinguen tres niveles de análisis del fenómeno cognitivo: las estructuras, los procesos y los estilos cognitivos. A continuación, se describen estos niveles de análisis según Zapata $\&$ Hernández (2014).

Las estructuras representan la manera como se organiza la información, según categorías, sistemas de constructos, sistemas causales y guiones o scripts. Esto, según un modo dinámico, se modifica constantemente cuando a través de las experiencias, contrastadas a la luz de los conocimientos previos, se genera nueva información o nuevos conocimientos. Las estructuras implican tanto a los esquemas de recuperación del conocimiento ya almacenado como a la incorporación de conocimiento nuevo. Por su lado, los procesos cognitivos aluden al procesamiento de la información y a 
la generación de conocimiento a partir de nueva información.

Tanto las estructuras como los procesos cognitivos están directamente determinados por los estilos cognitivos, que se relacionan con los aspectos particulares de la forma de pensar de cada individuo. Se puede hacer referencia al estilo cognitivo de un individuo y también al de una organización (como reunión de individuos) y esto determina a qué información se le presta mayor o menor atención, cuáles aspectos se consideran primordiales a la hora de tomar una decisión, cuáles estrategias se siguen para analizar la información de la que se dispone y qué importancia se asigna a la información que no está disponible (Blaylock \& Rees, 1984; Cheng, Luckett \& Schulz, 2003).

En este mismo orden de ideas, Zapata \& Hernández (2014) describen los estilos cognitivos como el sistema estructural de la personalidad y consideran que, al igual que las estructuras organizativas, son consistentes en el tiempo; por tanto, tienden a ser estables, no se modifican fácilmente en períodos cortos de tiempo. Así, los estilos cognitivos se convierten en sesgos o predisposiciones mentales.

\section{Los sesgos cognitivos y la toma de decisiones gerenciales}

El modelo clásico de elección racional para la toma de decisiones predominó en la economía hasta la década de los setenta. Este sostiene que el individuo elige la opción que represente una combinación óptima de probabilidad y utilidad, después de haber evaluado primero la probabilidad de cada resultado posible; luego, determinando la utilidad que se deriva de cada resultado; y, por último, combinando estas dos evaluaciones. Este cálculo combinado de probabilidad y utilidad puede ser un juicio bastante difícil de lograr en la práctica (Cortada \& Macbeth, 2006).

Simon (1947) apuntó las debilidades de este modelo de total racionalidad al señalarlo como poco realista para describir el juicio humano. Para esto, propuso un criterio más limitado para describir la realidad, que implica un proceso de toma de decisiones denominado racionalidad limitada. Este autor comienza por reconocer que en el proceso mental humano existen limitaciones inherentes; esto se refiere a que las personas eligen y razonan racionalmente, pero solamente dentro de las restricciones impuestas por sus conocimientos, sus capacidades de cálculo y su propia percepción. De esta forma, establece una distinción entre lo que denomina el hombre económico, que escoge siempre la mejor alternativa con el fin de maximizar los resultados, y el hombre administrativo, que recurre a simplificaciones de la complejidad del mundo real basado en sus propias limitaciones, lo que le permite escoger la mejor alternativa en función de la información restringida de que dispone y de sus propios juicios de valor al respecto.

Los aspectos fundamentales de esta distinción se pueden apreciar en la tabla 1, expresados como las diferencias entre la racionalidad objetiva asociada al hombre económico y la racionalidad limitada asociada al hombre administrativo.

Pero, la pregunta es ¡cómo se expresa la racionalidad limitada en el mundo organizacional? Para March \& Simon (1958), las organizaciones son un conjunto de individuos embarcados en un proceso de toma de decisiones. Estos individuos tienen sus propias necesidades, motivos e inclinaciones. Sus conocimientos sobre el mundo son limitados y, por tanto, también lo es su capacidad para aprender y solucionar problemas.

Simon oponía la racionalidad limitada al modelo clásico de la elección racional, aplicado al mundo de las organizaciones y la toma de decisiones gerenciales. En el campo de estudio de la psicología, Kahneman y Tversky (1974) desarrollan el estudio de los juicios humanos frente al riesgo y la incertidumbre e introducen un enfoque sobre heurísticos y sesgos cognitivos, también con el fin de desafiar 


\section{Tabla 1. Diferencias entre la racionalidad objetiva y la racionalidad limitada}

\begin{tabular}{|c|c|c|}
\hline & Racionalidad objetiva & Racionalidad limitada \\
\hline Conocimiento previo & $\begin{array}{l}\text { Debe ser completo y abarcar las con- } \\
\text { secuencias exactas de cada elección. }\end{array}$ & El conocimiento es fragmentario. \\
\hline Consideración de las consecuencias & $\begin{array}{l}\text { Se deben considerar todas las con- } \\
\text { secuencias que puede acarrear cada } \\
\text { elección. }\end{array}$ & $\begin{array}{l}\text { Las consecuencias son hechos futu- } \\
\text { ros. En muchos casos la imaginación } \\
\text { debe suplir a la falta de experiencia o } \\
\text { a las limitaciones del conocimiento. }\end{array}$ \\
\hline Elección & $\begin{array}{l}\text { Se basa en la consideración de todos } \\
\text { los posibles comportamientos alter- } \\
\text { nativos. }\end{array}$ & $\begin{array}{l}\text { Se basa en la consideración de unas } \\
\text { pocas de estas posibles alternativas. }\end{array}$ \\
\hline
\end{tabular}

Fuente: Elaboración propia basada en el autor Simon (1947).

los modelos que dominaban en la época y que eran estrictamente racionales, desarrollando así su propia perspectiva de la racionalidad limitada. El enfoque de estos autores generó investigaciones en el campo de la psicología que se han extendido al saber académico en Economía, Derecho, Sociología, Medicina y Ciencias políticas (Cortada, 2008). Su obra se ha desarrollado a partir de la idea de estudiar los aspectos que determinan la toma de decisiones en situaciones en que los beneficios y las pérdidas son inciertos (Chahin, 2016).

Es así como Daniel Kahneman, psicólogo de profesión, fue galardonado en el año 2002 con el premio Nobel de Economía, por sus estudios sobre la toma de decisiones en situaciones de riesgo e incertidumbre, pasando de ser catedrático de Psicología de la Universidad de Princeton a miembro de la Real Academia de Ciencias Económicas y Financieras (Chahin, 2016). Según este autor, las contribuciones de Kahneman y sus colaboradores a la administración pueden resumirse a través de una revisión de sus principales publicaciones, como se muestra en la tabla 2.

Las ideas desarrolladas en la tabla 2 describen las condiciones bajo las cuales se realizan los procesos de toma de decisiones en la organización. El individuo, o el equipo encargado de una decisión, se desenvuelve dentro de los límites de la racionalidad, pues tiene una visión parcial y subjetiva de la situación. Adicionalmente, posee patrones de pensamiento que predisponen la forma como analiza y resuelve problemas, conocidos como los sesgos cognitivos, desarrollados bajo los postulados de la teoría cognitiva. Estudiar estos patrones de pensamiento permite entender, en parte, cómo las personas piensan y deciden.

Schwenk (1984, p. 111) define las decisiones estratégicas "como una clase especial de toma de decisiones bajo incertidumbre. Tales decisiones implican las actividades de formulación de objetivos, identificación de problemas, generación de alternativas y evaluación/selección”. Duhaime \& Schwenk (1985) indican que las decisiones estratégicas se caracterizan fundamentalmente por su falta de exactitud en su estructura, y esto se debe a la complejidad de los problemas estratégicos. Esta complejidad se explica porque el problema estratégico no tiene una clara formulación, es difícil describir el problema y determinar los criterios por los cuales los individuos deben guiarse para juzgar las soluciones adecuadas (Schwenk, 1984).

Así, los investigadores de la psicología cognitiva y los teóricos de las decisiones del comportamiento behavioural decision theorists - han identificado una 


\section{Tabla 2. Aportes de Kahneman al estudio de la administración}

\begin{tabular}{|c|c|c|}
\hline Aporte & Autores/año & Descripción \\
\hline $\begin{array}{l}\text { Aversión de la pérdi- } \\
\text { da de elección libre } \\
\text { de riesgo }\end{array}$ & $\begin{array}{l}\text { Tversky y Kah- } \\
\text { neman (1991) }\end{array}$ & $\begin{array}{l}\text { Se basa en la hipótesis de que las pérdidas y las desventajas tienen mayor im- } \\
\text { pacto en las preferencias de las personas que las ganancias y las ventajas. La } \\
\text { elección que ejerce una persona depende del nivel de statu quo o referencia, } \\
\text { es decir, que los cambios del punto de referencia suelen llevar a revertir la } \\
\text { preferencia. }\end{array}$ \\
\hline $\begin{array}{l}\text { Bajas probabilidades } \\
\text { de utilidad }\end{array}$ & $\begin{array}{l}\text { Kunreuther, No- } \\
\text { vemsky y Kah- } \\
\text { neman (2001) }\end{array}$ & $\begin{array}{l}\text { Estudia cómo las personas procesan la información sobre eventos negativos } \\
\text { con alta probabilidad de ocurrencia. Concluyen que las personas necesitan } \\
\text { mucha información del contexto en el que ocurren los hechos para poder } \\
\text { juzgar las diferencias entre los riesgos sometidos a su consideración. Para que } \\
\text { realmente se valore el riesgo en toda su dimensión, debe encontrarse dentro } \\
\text { de la escala de probabilidad percibida por las personas. }\end{array}$ \\
\hline $\begin{array}{l}\text { Mapas de racionali- } \\
\text { dad limitada }\end{array}$ & $\begin{array}{l}\text { Kahneman } \\
(2002)\end{array}$ & $\begin{array}{l}\text { Explora la psicología de las creencias y las opciones intuitivas, y examina su } \\
\text { racionalidad limitada. Presenta un análisis de los heurísticos del juicio, la } \\
\text { elección arriesgada y los efectos que producen. Estudia las intuiciones, los } \\
\text { pensamientos y las preferencias que vienen a la mente de inmediato, sin tiem- } \\
\text { po para la reflexión. Analiza la noción de que los pensamientos se diferencian } \\
\text { en una dimensión de la accesibilidad. Esto significa que hay algunos que } \\
\text { vienen a la mente mucho más fácil que otros. Además, plantean la distinción } \\
\text { entre los pensamientos intuitivos y los deliberados. }\end{array}$ \\
\hline $\begin{array}{l}\text { Los sesgos en la toma } \\
\text { de decisiones }\end{array}$ & $\begin{array}{l}\text { Kahneman, Lo- } \\
\text { vallo y Sibony } \\
\text { (2011) }\end{array}$ & $\begin{array}{l}\text { Estudian el impacto de los sesgos sobre las decisiones estratégicas, planteando } \\
\text { formas de detectarlos. Relacionan el concepto de los sesgos con posibles fallas } \\
\text { del individuo al momento de tomar decisiones, haciendo énfasis en las fallas } \\
\text { relacionadas con la percepción. Además de estas fallas, introducen otras cau- } \\
\text { sas que, aunque no están directamente relacionadas con los sesgos, influyen } \\
\text { a la hora de la toma de decisiones. Resaltan la importancia de la economía } \\
\text { conductual a la hora de tomar decisiones, retomando la idea original de los } \\
\text { sesgos cognitivos y su impacto en la toma de decisiones. }\end{array}$ \\
\hline \multirow[t]{3}{*}{$\begin{array}{l}\text { Pensar rápido, pensar } \\
\text { despacio }\end{array}$} & $\begin{array}{l}\text { Kahneman } \\
(2012)\end{array}$ & $\begin{array}{l}\text { Presenta de qué manera trabaja la mente, teniendo en cuenta los más recien- } \\
\text { tes avances en psicología cognitiva y social. Define dos vías de pensamiento: } \\
\text { el Sistema 1, rápido, intuitivo y emocional; y el Sistema } 2 \text {, más lento, esfor- } \\
\text { zado y racional. }\end{array}$ \\
\hline & & $\begin{array}{l}\text { El Sistema 1: es intuitivo, opera sin ningún esquema de pensamiento, es lo } \\
\text { primero que viene a la mente. }\end{array}$ \\
\hline & & $\begin{array}{l}\text { El Sistema 2: es racional, tiene asociada una actividad mental completa y un } \\
\text { trabajo de análisis. Una de sus funciones es observar y controlar las acciones } \\
\text { planteadas por el Sistema } 1 \text {. Se han hecho investigaciones para determinar } \\
\text { si hay relación entre el pensamiento y el autocontrol. Estas investigaciones } \\
\text { han permitido clasificar a las personas por su autocontrol y su capacidad } \\
\text { cognitiva. }\end{array}$ \\
\hline
\end{tabular}

Fuente: Elaboración propia Basada en el autor Chahin (2016). 
serie de heurísticos y sesgos cognitivos a los cuales están sujetos los individuos en sus procesos de juicios o toma de decisiones bajo situaciones de incertidumbre y complejidad (Tversky \& Kahneman, 1973, 1974; Makridakis, 1990; Kahneman, Slovic \& Tversky, 1982; Das \& Teng, 1999; Fisk, 2002; Vidar \& Lechner, 2013; Otuteye \& Siddiquee, 2015). Al respecto, Duhaime \& Schwenk (1985) sugieren que los individuos responsables de tomar decisiones estratégicas en las organizaciones pueden usar los procesos de simplificación estratégica o sesgos cognitivos.

Los sesgos cognitivos, según Zapata \& Canet (2009), son reglas de juicio de las cuales dependen los encargados de tomar decisiones, que les ayudan a simplificar las situaciones complejas y así poder definir los modelos de decisiones y las acciones más convenientes. Estos investigadores resumen, una vez abordada una serie de referencias con respecto al tema, lo siguiente:

Los sesgos cognitivos permiten desarrollar modelos simplificados del mundo o de la realidad que rodea a los directivos y gerentes, con el propósito de facilitar tanto el proceso de toma de decisiones bajo situaciones complejas como el desarrollo de propuestas de solución a los problemas empresariales; es decir, permiten reducir las difíciles tareas mentales a tareas más sencillas, y entre estas la definición de la estructura organizativa de la empresa y de sus variables de diseńo asociadas. (Zapata \& Canet, 2009, p.244) ${ }^{1}$.

Katz (1992), en su trabajo clásico -donde desarrolla una propuesta de modelo cognitivo psicosocial de elección-, reconoce que los procesos heurísticos contribuyen a destapar la caja negra acerca de la manera como las personas hacen sus elecciones o

1. Busenitz \& Barney (1997) tienen una visión positiva de los heurísticos y los sesgos cognitivos. En este sentido, manifiestan que proveen una manera adecuada para aproximarse a las decisiones adecuadas. decisiones individuales. Por su parte, Kahneman et al. (2011) afirman que la influencia de los sesgos cognitivos se hace menos intensa al pasar de lo individual a lo grupal. Mientras que es muy difícil para un decisor detectar sus propios sesgos cognitivos, estos pueden tornarse más evidentes cuando se integra a un grupo dentro de la organización, responsable de tomar alguna decisión importante. Afirman estos autores que los sesgos que no pueden verse en sí mismos se hacen visibles en los pares, es por esta razón que resulta favorecedor que las decisiones sean tomadas por varias personas. Esta posición coincide con la de Schneider \& Angelmar (1993), quienes plantean que el modelo cognitivo para el análisis organizativo debe estar orientado fundamentalmente al estudio de la cognición a nivel de grupo, organizativo e inter-organizativo, considerando siempre las relaciones de la cognición con la acción y el desempeño organizacional.

Dentro del marco de las ideas anteriores, cabe destacar lo afirmado por Liu (2017) en cuanto a que la cognición compartida durante los procesos organizacionales se constituye en un factor importante en la toma de decisiones de los equipos de alta gerencia (TMT). Esto se debe a que esta cognición refleja la comprensión y el acuerdo en la toma de decisiones con respecto a las metas y las estrategias organizacionales; y que, además, la cognición compartida no significa que no haya heterogeneidad ni conflicto, sino que aquella más bien contribuye a su resolución mediante la interacción efectiva y la comunicación entre los diferentes miembros, lo que trae consigo mejoras en la eficiencia de los equipos.

La premisa del beneficio de la decisión grupal está directamente relacionada con otro de los aportes descritos en la tabla 4, referida a los dos sistemas de pensamiento presentados por Kahneman (2012) en su libro Pensar rápido, pensar despacio. En esta obra, el autor describe dos sistemas de pensamiento, uno intuitivo, identificado como sistema de pensamiento 1 , y otro analítico, identificado 


\section{Tabla 3. Tipos de predisposiciones (sesgos cognitivos) en la toma de decisiones}

\begin{tabular}{ll}
\hline \multicolumn{1}{c}{ Tipo de sesgo } & \multicolumn{1}{c}{ Descripción } \\
\hline Razonar por analogía & Aplicar la analogía implica proveer una imagen de lo que puede ser utilizado para \\
& la comprensión, definición y solución de un problema. Por ejemplo, un negocio \\
& parecido puede ser adquirido puesto que los directivos, por analogía, lo consideran \\
& conocido.
\end{tabular}

Fuente: Adaptado de Makridakis (1990); Duhaime \& Schwenk (1985); Kahneman, Slovic \& Tversky (1982).

como sistema de pensamiento 2, que coexisten e interactúan constantemente en la mente humana. Al aplicar la noción de estos dos sistemas a las circunstancias que implica una decisión en grupo, Kahneman et al. (2011) afirman que el sistema de pensamiento 2 permite identificar errores en las recomendaciones dadas por otros en el uso del sistema de pensamiento 1 .

2. Simon \& Houghton (1999) y Simon, Hougton \& Aquino (2000) señalan que el sesgo de ilusión de control lleva al individuo a creer controlar, en gran parte, eventos incontrolables, conduciéndoles a predecir con exactitud los resultados de tales eventos.
Numerosos autores, entre ellos los precursores del enfoque, Kahneman \& Tversky (1974), hacen distinción entre los términos heurísticos y los sesgos cognitivos, resaltando que los heurísticos son los principios que se emplean para evaluar y para predecir valores, y los sesgos cognitivos están asociados a estos heurísticos. Pero existe otra tendencia, como la adoptada, por ejemplo, por Schwenk (1984) y Busenitz \& Barney (1997), que utilizaron en sus investigaciones los dos términos en forma indistinta. En función de estos planteamientos, en la presente investigación se asume la posición de usar el término de sesgos cognitivos. En la tabla 3 se muestran algunos sesgos cognitivos. 
Por su parte, Urra Medina \& Acosta (2011) afirman que los sesgos cognitivos parecen ser interculturales y resistentes al conocimiento. Los definen como aquellos procesos normativos simplificadores de selección, procesamiento y ajuste de la información que conducen a sesgos de valoración y predicción, entendiéndose estos desde su connotación negativa o de desviación. Esta visión de los sesgos cognitivos como causantes de decisiones más ajustadas al pensamiento de los decisores que a las condiciones reales que las motiva, les confieren la propiedad de ser potencialmente distorsionantes y ocasionar efectos negativos en la organización. Por tal motivo, estos autores resaltan la atención que la gerencia debe otorgar a los sesgos cognitivos y la importancia de que los directivos se enfoquen en contribuir a su comprensión y gestión, ya que el conocimiento de cómo operan y de las consecuencias que acarrean permite gestionar los factores de contingencia que pueden ser configurados $y$, por tanto, controlados, como medida para prevenir los efectos perniciosos en los procesos organizativos de adopción de decisiones.

Al respecto, Kahneman et al. (2011) consideran que, aunque se han hecho estudios que permiten identificar y describir diferentes sesgos que influyen en el comportamiento de los gerentes, solo conocerlos y describirlos no permite contrarrestar los posibles efectos negativos que una mala decisión empresarial puede acarrear, por lo que proponen tomar medidas al respecto. Para apoyar esta idea, los autores hacen referencia a un estudio de McKinsey Quarterly, publicado en marzo de 2010, basado en la revisión de más de 1,000 inversiones en grandes empresas. Los resultados mostraron que cuando las organizaciones tomaron medidas para reducir el efecto de los sesgos cognitivos en los procesos de toma de decisiones lograron rendimientos de hasta siete puntos porcentuales más altos.

En esta publicación, los autores desarrollan una estrategia basada en una lista de chequeo de 12 preguntas, con el fin de hacer visibles los sesgos de un equipo de toma de decisiones en una organización. Las preguntas están divididas en tres categorías: preguntas que los tomadores de decisiones deben hacerse a sí mismos, preguntas que deben usar para desafiar a la gente que propone un curso de acción, y las preguntas de evaluación de la propuesta. Este tipo de herramientas permite dar una utilidad concreta a una idea abstracta y relacionada con aspectos complejos de la cognición, como son los sesgos cognitivos. Hacer visible su existencia, conceptualizarlos, conocer sus implicaciones y diseñar herramientas para aplicar en los diferentes ámbitos de la organización puede resultar de gran utilidad para la alta gerencia.

Montibeller \& VonWinterfeldt (2015) hacen una distinción entre sesgos difíciles y sesgos fáciles de corregir. Afirman que los sesgos difíciles son resistentes a la lógica, a la descomposición y al uso de herramientas de entrenamiento. Entre estos sesgos mencionan el exceso de confianza y el anclaje. Entre los sesgos que los autores consideran fáciles de corregir, a través de herramientas como el uso de datos estadísticos, la probabilidad y la lógica, está la correlación ilusoria.

\section{Los sesgos cognitivos y la percepción de los gerentes}

Partiendo de las ideas desarrolladas hasta ahora, los procesos gerenciales implican, básicamente, tomar decisiones estratégicas que apuntan a aspectos diversos de la organización, desde el diseño de estructuras y procesos hasta aspectos como la cultura organizacional y las relaciones con los grupos de interés. Zapata, Mirabal \& Canet (2015) describen el entorno organizacional como competitivo y exponen que está representado por oportunidades y amenazas a las que la organización debe ajustarse, equilibrando las variables internas de diseño organizativo $^{3}$ con las variables externas del entorno

3. El diseño organizativo se refiere al conjunto de actividades, acciones y decisiones estratégicas y operativas que toman los di- 
donde se desenvuelve. Según estos autores, este ajuste:

Depende en gran medida de la percepción e interpretación de los directivos y gerentes sobre esos condicionantes. Es decir, la traducción de los eventos del entorno conduce al desarrollo de modelos y esquemas conceptuales que permiten comprenderlo y, en consecuencia, poner en práctica soluciones a determinados problemas a los que la organización se enfrenta. (Zapata et al. 2015, p. 787)

Esta percepción e interpretación de los directivos y los gerentes se traduce en lo que Weick (1969) describe como el papel de intermediarios que estos representan entre el medio ambiente y los efectos que este produce en el interior de la organización. "Esto significa que los criterios de selección tienen su origen más bien en quienes toman las decisiones y no en el medio ambiente" (Weik, 1969, p. 127). Por tanto, el entorno, más que una representación objetiva de la realidad es el resultado de la visión de quienes lo perciben. En este sentido, Weick (1969) señala que las organizaciones reconocen al entorno mediante el proceso de percepción de los directivos y que, por tanto, es el producto de una ordenación abreviada, generalizada y corregible de las distintas experiencias dentro de la psiquis que sirven de marco de referencia inicial para la acción. Zapata et al. (2015, p. 791) aclaran: "Es decir, la gerencia activamente selecciona o crea el entorno dónde quiere participar, competir y estructurar las decisiones necesarias para ajustar sus percepciones acerca del mundo que los rodea".

Para estos autores, la percepción del entorno es un proceso cognitivo que sirve de guía orientadora

rectivos y los gerentes con el propósito de definir los procesos, la estructura y las formas de organización que adopta la empresa en un momento determinado y bajo ciertas condiciones contextuales a las que tiene que adaptarse para poder alcanzar sus metas y objetivos (Zapata \& Hernández, 2014). para el proceso de toma de decisiones estratégicas que contribuyen a la definición de la estructura organizativa y los procesos organizacionales, así como a la elección de los factores ambientales con mayor impacto en su comportamiento, según esa percepción. Esto favorece, por ende, la formación de la visión directiva, vinculada con la forma como debe ser gestionada la empresa. Resaltan estos autores que uno los aspectos que afecta la percepción del entorno es la incertidumbre, que se constituye en uno de los principales problemas a enfrentar y resolver por la dirección de la empresa. Al respecto, Schwenk (1988) muestra un interés especial sobre el proceso cognitivo de percepción, puesto que es fundamental en el estudio que enlaza el entorno, la estrategia y la estructura de la organización.

Por su parte, Wilson, Centerbar \& Gilbert (2005) afirman que la incertidumbre es una de las razones que causa a la mente humana mayor debilitamiento por ansiedad y, en consecuencia, es un objetivo primordial eliminarla o al menos disminuirla. La manera como las personas enfrentan los hechos de su vida diaria depende de la imagen creada por sus puntos de vista y las teorizaciones sobre cómo desplazarse de un estado de ignorancia a uno de conocimiento, con el fin de reducir la incertidumbre e incrementar la predictibilidad (Bahmani, Reza \& Hamidi, 2015).

Milliken (1987, p. 136) define la incertidumbre del entorno como "la incapacidad de un individuo de predecir algo con exactitud, debido a que siente la carencia de información suficiente para pronosticar eventos o a la poca capacidad para discriminar entre datos relevantes e irrelevantes". Esta definición de incertidumbre del entorno guarda estrecha relación con el concepto de racionalidad limitada descrito anteriormente, en cuanto a la visión parcial de la realidad; también se vincula con los sesgos o juicios de valor que determinan la perspectiva de la toma de decisiones al momento de tratar de enfrentar y simplificar las situaciones de alta complejidad. 
Tabla 4. Los sesgos cognitivos y los sistemas de contabilidad y control de gestión

\section{Nombre del artículo}

Relación entre los sistemas de contabilidad y control de gestión y los sesgos en la evaluación y toma de decisiones Facultad de Ciencias Económicas:

Investigación y Reflexión

Aspectos relevantes

Objetivo: contribuir con la literatura de control de gestión aportando un marco conceptual que permita una mejor comprensión de la relación entre los Sistemas de Contabilidad y Control de Gestión (SCCG) y la existencia de sesgos en las evaluaciones y la toma de decisiones.

Medición de sesgos: se relacionaron dos variables: las características del usuario (limitaciones cognitivas, aspectos motivacionales) con las características de los SCCG (presentación de la información, grado de subjetividad). Luego, establecieron los juicios presentes en esta relación, clasificándolos como beneficiosos o disfuncionales. Las características de los SCCG se consideran como las variables independientes y los sesgos cognitivos como las variables dependientes. En función de esta información se elaboraron matrices relacionales.

Conclusiones: los diseñadores de los SCCG desempeñan un papel determinante en la objetividad y neutralidad de estos, ya que tanto sus limitaciones cognitivas como sus aspectos motivacionales pueden condicionar el diseño de estas herramientas, causando obstáculos en las evaluaciones, así como decisiones sesgadas.

Fuente: elaboración propia basada en los autores López, Gómez \& Naranjo (2016).

\section{Tabla 5. Los sesgos cognitivos y el Derecho}

Nombre del artículo
Los sesgos cognitivos y el Derecho: el influjo de lo irracional

Fuente: elaboración propia basa en Muñoz (2012).

\section{Aplicación de la teoría cognitiva en el campo de} la organización

Una revisión de la literatura permite observar la importancia creciente del estudio de los sesgos cognitivos y su aplicación en campos tan diversos como el Derecho, la Administración de Salud, opciones terapéuticas en psicología, discriminación laboral, toma de decisiones gerenciales, entre otros. Este interés de la comunidad científica apunta a que el tema se seguirá desarrollando en el futuro. A continuación, una breve revisión de algunos trabajos 
Tabla 6. Exceso de confianza y optimismo en las decisiones de presupuesto de capital

\section{Nombre del artículo}

Exceso de confianza y optimismo en las decisiones de presupuesto de capital: las finanzas corporativas desde un enfoque centrado en el comportamiento

\section{Autor}

Useche (2014)

Aspectos relevantes

Objetivo: analizar comparativamente el proceso de evaluación de decisiones de inversión, desde la teoría financiera tradicional y desde un nuevo enfoque complementario que surge de las finanzas corporativas basadas en el comportamiento (Behavioral Corporate Finance).

Medición de sesgos: se realiza a través de una revisión teórica de la literatura científica referida a los sesgos de exceso de confianza y optimismo.

Conclusiones: la eliminación de los sesgos en el comportamiento es, a la vez, imposible e indeseable. Lo más conveniente es que la empresa reconozca la existencia de influencias del comportamiento en sus decisiones financieras, identifique sus impactos positivos y negativos e introduzca estos elementos explícitamente en su toma de decisiones.

Fuente: elaboración propia basada en Useche (2014).

que muestran la aplicación de la teoría cognitiva de la organización, específicamente en el aspecto de los sesgos cognitivos.

Adicionalmente, cada vez son más comunes las prácticas orientadas a disminuir los efectos negativos de los sesgos cognitivos en los procesos organizacionales. Algunas de estas prácticas, aunque no provienen de la literatura científica, no dejan de ser una señal del reconocimiento a la importancia creciente del tema para la alta gerencia. Un ejemplo de esto son las sesiones de Unconscious Bias (sesgo inconsciente) que se realizan cada vez con más frecuencia en el Reino Unido. Estas sesiones están orientadas a posicionar la creación de una cultura de inclusión en la empresa, como un esfuerzo colectivo para fomentar la igualdad y la no discriminación y, al mismo tiempo, evitar prejuicios inconscientes hacia determinados colectivos. Algunos trabajos como el de Ross (2008), titulado Proven Strategies for Addressing Unconscious Bias in the Workplace, proveen a los ejecutivos y gerentes de herramientas dirigidas a reconocer los sesgos y evitar la discriminación dentro de las organizaciones, alineándolas con metas globales como la igualdad de género y la inclusión. Este trabajo es una publicación bajo el patrocinio de una ONG estadounidense denominada Diversity Best Practices, que, a su vez, forma parte de una iniciativa para brindar soporte a madres trabajadoras.

La importancia de los sesgos cognitivos ha trascendido la gestión de personas y se extiende a muchas decisiones de negocio. Pensar en las empresas como productoras de decisiones apunta a otros aspectos que pueden verse afectados por los sesgos, como el control de calidad. En este orden de ideas, en el campo empresarial cada vez son más comunes los esfuerzos para diseñar estrategias y contrarrestar los efectos adversos de los sesgos cognitivos. Klein (2007) afirma que una de las razones por las que un alto número de proyectos falla es porque algunas personas se resisten a expresar sus reservas durante la fase de planeación. Según este autor, las llamadas técnicas de oportunidad de éxito representan una herramienta valiosa para hacerlos sentir seguros de disentir, basados en el conocimiento del proyecto o en alguna duda sobre la debilidad de la idea. Una de estas herramientas prácticas dentro de las técnicas de oportunidad de éxito es lo que se conoce como 
Pre-mortem exercise, definido por este autor como un ejercicio de visualización que consiste en pedir a los ejecutivos participantes en una gran decisión estratégica que se proyecten en el futuro e imaginen y escriban por qué el proyecto ha fracasado. Esto permite que, aunque la decisión se mantenga, se incluyan medidas para minimizar los riesgos.

\section{Reflexiones finales}

La psicología cognitiva analiza las estructuras y el funcionamiento de los procesos mentales con el fin de comprender la conducta humana. Sus postulados se afianzan en teorías sobre la atención, la memoria, la percepción, la emoción, los procesos del pensamiento y la resolución de problemas y su aplicación. Los trabajos de Neisser (1967) en el campo de la psicología cognitiva se aceptan como los pioneros de esta disciplina.

La psicología cognitiva reconoce que el conocimiento de la realidad se adquiere a través de estímulos sensoriales que son procesados por un sistema mental complejo que involucra las siguientes funciones: la percepción, la atención, el pensamiento, la memoria y el lenguaje. March y Simon estudiaron las características del comportamiento humano en las organizaciones, partiendo de los postulados principales de la psicología cognitiva. De la aplicación de estos principios al ámbito gerencial surge, dentro de la teoría del management estratégico, la denominada teoría cognitiva de la organización, cuyos fundamentos se recogen en una serie de investigaciones que se enfocan en estudiar la mente del directivo, específicamente en la formación de la estrategia y su relación con los procesos de toma de decisiones. Esta teoría pone especial atención en cómo los procesos cognitivos afectan todos los ámbitos de acción de la organización, desde las variables de diseño organizativo hasta la forma como la organización percibe y responde a las condiciones de su entorno.
Desde esta perspectiva, el management es básicamente un proceso cognitivo de toma de decisiones en condiciones de complejidad e incertidumbre, que implica acciones como buscar dar significado a situaciones confusas partiendo de información ambigua, escasa e incompleta.

La comprensión de los procesos cognitivos, según sus tres niveles de análisis: las estructuras, los procesos cognitivos y los estilos cognitivos, permite determinar los aspectos particulares de la forma de pensar de cada individuo. Se puede hacer referencia al estilo cognitivo de un individuo y también al de una organización (como reunión de individuos). Los estilos cognitivos influyen directamente, tanto en las estructuras como en los procesos, dotándolos de características propias y distintivas en cada individuo u organización; de ahí que diferentes estilos cognitivos pueden generar diferentes estrategias organizacionales (Mintzberg et al., 1999).

Los estilos cognitivos son considerados una parte importante del sistema estructural de la personalidad y contribuyen a definir patrones a través de los cuales se simplifica la complejidad de la realidad para compensar la visión parcial de los hechos sobre los cuales los gerentes deben decidir; con esto se pretende reducir la ansiedad por los riesgos que lleva implícita la incertidumbre. Estos patrones, conocidos como sesgos cognitivos, pueden ser considerados como condicionantes de la racionalidad limitada que determina el esquema de actuación de los individuos dentro de la organización.

La racionalidad limitada es entendida como la restricción en la cantidad de información sobre la realidad de la que un individuo puede disponer y que es capaz de procesar, restricción relacionada directamente con las características propias de su cognición. Para superar esta restricción, el pensamiento recurre a los sesgos cognitivos que sirven como atajos para simplificar y compensar los efectos de su visión incompleta y, por tanto, imprecisa. Estos atajos del pensamiento o sesgos cognitivos 
están relacionados estrechamente con la toma de decisiones.

En el ámbito empresarial, las decisiones involucran aspectos vitales para la organización que se pueden traducir en ventajas o desventajas competitivas, llegando incluso a comprometer la supervivencia. Decidir involucra analizar situaciones inciertas, con las limitaciones de la información de la que se dispone, según los sesgos cognitivos del decisor. En esta investigación se asume la posición de que los sesgos son potencialmente distorsionantes; por tal razón, pueden tener una connotación negativa en un proceso de toma de decisiones. La posición de la mayoría de los autores de esta corriente se concentra en desarrollar estrategias orientadas a minimizar su impacto.

Tal como se ha expresado en párrafos precedentes, un sector de la comunidad científica orientada a estudiar la organización, desde el punto de vista de sus procesos cognitivos, reconoce en los sesgos cognitivos un potencial distorsionante. Reconocer la existencia de los sesgos es el primer paso para iniciar un proceso de revisión y estudio de las características de estos, que permita su comprensión e identificación en los decisores de la organización, entendiendo que el estilo de pensamiento de una organización está directamente determinado por la combinación de los sesgos cognitivos de los individuos encargados de conducirla; por tal motivo, el estudio de los sesgos cognitivos se considera de gran importancia dentro de la teoría de la organización.

Estudios serios en el área se han enfocado en definir los principales sesgos que pueden afectar el proceso de toma de decisiones de una organización, asociados a los posibles efectos que pueden ocasionar, lo que se considera un insumo importante y valioso para la gerencia. El conocimiento de cómo operan y las consecuencias que acarrean permite gestionar los factores de contingencia que pueden ser configurados y controlados como medida para prevenir los efectos perniciosos en los procesos organizativos de toma de decisiones. De este modo, los equipos de decisión se consideran una herramienta valiosa para contrarrestar las posibles distorsiones de los sesgos, esto basado en la idea de que resulta difícil reconocer los sesgos propios, mientras que se hace más sencillo reconocerlos en otros.

Otro de los aspectos que aparece frecuentemente relacionado con los sesgos es la percepción del entorno por parte de los gerentes y los directivos. Según la perspectiva cognitiva, el entorno no es un conjunto de condiciones totalmente reales y objetivas, sino que más bien es el resultado de la percepción que los directivos y los gerentes tienen sobre las oportunidades y las amenazas que este representa. Se considera que uno de los factores principales que afecta la percepción del entorno es la incertidumbre, por lo que se reconoce que los sesgos cognitivos pueden proporcionar vías para contrarrestar los efectos del estrés que esta puede causar, haciendo parecer los hechos más predecibles $y$, por tanto, más controlables de lo que son en realidad.

El estudio de los sesgos cognitivos ha sido aplicado en distintas áreas del conocimiento y se ha orientado principalmente a entender cómo y por qué los individuos deciden de determinada manera. Este conocimiento se ha utilizado en el análisis de aspectos tan disímiles como: el diseño de sistemas de contabilidad y control de gestión, derecho, juegos de azar, presupuestos de capital, diagnósticos médicos, entre otros. Existen numerosos ejemplos en la literatura científica de diseños, tanto teóricos como experimentales, que tienen como fin realizar la medición de sesgos cognitivos, mostrando el potencial de la perspectiva cognitiva para predecir, aunque sea de manera aproximada, el comportamiento de la alta gerencia y así intentar anticipar los efectos de estos comportamientos en todas las áreas del desempeño de la organización.

Son muchas las posibilidades de estudio que la teoría cognitiva de la organización ofrece al management estratégico; posibilidades que van desde herramientas para comprender y facilitar la toma de decisiones bajo condiciones de complejidad e incertidumbre 
hasta la identificación de patrones que permitan categorizar a los decisores según las áreas de la organización donde se desenvuelvan o según algunos aspectos estratégicos como: la gestión del conocimiento, los procesos de internacionalización, el establecimiento de asociaciones y redes de cooperación, el diseño de la estructura organizativa y el desarrollo de los procesos organizacionales, entre otros.

\section{Referencias}

Bahmani, Z., Reza, M., \& Hamidi, M. (2015). Investigation of manager's perceived uncertainty and environmental scanning behavior in second industrial area of ahvaz, Iran. International journal of scientific management and development, 3(5), 334-340.

Banyard, P., Cassells, A., Green P., Hartland, J., Hayes, N. \& Reddy, P. (1995). Introducción a los procesos cognitivos. Barcelona. Ariel Psicología.

Blaylock, B. \& Rees, L. (1984). Cognitive style and the usefulness of information. Decision Sciences, 15(1) 74-91.

Busenitz, L. \& Barney, J. (1997). Differences between entrepreneurs and managers in large organizations: Biases and heuristics in strategic decision-making. Journal of Business Venturing, 12(1), 9-30.

Chahin, T. (2016). El impacto de los trabajos de Daniel Kahneman en la administración. Ciencias Administrativas, 4(7), 49-56.

Cheng, M., Luckett, P., \& Schulz, A. (2003). The effects of cognitive style diversity on decision-making dyads: An empirical analysis in the context of a complex task. Behavioral research in accounting, 15(1), 39-62.

Cortada, N. (2008). Los sesgos cognitivos en la toma de decisiones. International journal of psychological research, 1(1), 68 -73.
Cortada, N. \& Macbeth, G. (2006). Los sesgos cognitivos en la toma de decisiones. Revista de Psicología UCA, 2(3), 55-71.

Escobedo, H. (1993). Psicología cognitiva. Informática educativa, 6(2), 167-173.

Das, T. K. \& Teng, B. S. (1999). Cognitive biases and strategic decision processes: An integrative perspective. Journal of Management, 36(6), 757-778.

Duhaime, I. \& Schwenk, C. (1985). Conjectures on cognitive simplification in acquisition and divestment decision making. Academy of Management Review, 10(2), 287-295.

Fisk, J. E. (2002). Judgments under uncertainty: Representativeness or potential surprise? British Journal of Psychology, 93(4), 431-449.

Gardner, H. (1987). Nueva ciencia de la mente. México: Paidós.

Kahneman, D. (2002). Maps of bounded rationality: A perspective on intuitive judgment and choice. Nobel prize lecture, 8, 351-401.

Kahneman, D. (2012). Pensar rápido, pensar despacio. Barcelona. Debate.

Kahneman, D., Lovallo D., \& Sibony O. (2011). Before you make that big decision. Harvard business review, 89(6), 51-60.

Kahneman, D., Slovic, P., \& Tversky, A. (1982). Judgment under uncertainty: Heuristics and biases. Cambridge: Cambridge University Press.

Kahneman, D. \& Tversky, A. (1974) Judgement under uncertainty: Heuristics and biases. Science, 185(27), 1124-1131.

Katz, J. (1992). Psychosocial cognitive model of employment status choice. Entrepreneurship Theory and Practice, 17(1), 29-37.

Klein, G. (2007). Performing a project premorten. Harvard business review, 85(9), 18-19. 
Kunreuther, H., Novemsky, N., \& Kahneman, D. (2001). Making low probabilities useful. Journal of risk and uncertainty, 23(2), 103-120.

López, E.; Gómez, J. \& Naranjo, D. (2016). Relación entre los sistemas de contabilidad y control de gestión y los sesgos en la evaluación y toma de decisiones. Facultad de Ciencias Económicas: Investigación y Reflexión, 24(1), 13-24.

Liu, Y. (2017). A review of the relationship between TMT shared cognition and strategic decision making. American Journal of Industrial and Business Management, 7(10), 1101-1110.

Makridakis, G. (1990). Forecasting, planning, and strategy for the $21^{\text {st }}$ century. New York: The free press.

March, J. \& Simon, H. (1958). Organizations. [Las citas y referencias corresponden a la versión en castellano: "Teoría de la Organización" (1987)]. Madrid: Ariel Economía.

Milliken, F. (1987). Three types of uncertainty about the environment: State, effect and response uncertainty. Academy of Management Review, 12(1), 133-143.

Mintzberg, H., Ahlstrand, B. \& Lampel, J. (1999). Safari a la estrategia: una visita guiada por la jungla del management estratégico. Primera edición. Argentina: Ediciones Granica S.A.

Montibeller, G. \& vonWinterfeldt, D. (2015) Cognitive and motivational biases in decision and risk analysis. Risk analysis, 35(7), 1230-1251.

Muñoz, A. (2012). Los sesgos cognitivos y el derecho: el influjo de lo irracional. El notario del siglo XXI, 42(2), 60-67.

Neisser, U. (1967). Cognitivepsychology. Englewood Cliffs: Prentice-Hall.

Nonaka, I (1994). A dynamic theory of organizational knowledge creation. Organization Science, 5(1), 14-37.
Otuteye, E. \& Siddiquee, M. (2015). Overcoming cognitive biases: A heuristic for making value investing decisions. The Journal of Behavioral Finance, 16(2), 140-149.

Riviere, A. (1991). Orígenes históricos de la psicología cognitiva: paradigma simbólico y procesamiento de la información. Anuario de psicología Universidad de Barcelona, 57(1), 129-155.

Ross, H. (2008). Proven strategies for addressing unconscious bias in the workplace. CDO Insights, 2(5), 1-18.

Schneider, S. \& Angelmar, R. (1993). Cognition in organizational analysis: Who's minding the store. Organization Studies, 14(3), 347-374.

Schwenk, C. (1984). Cognitive simplification processes in strategic decision-making. Strategic management journal, 5(2), 111-128.

Schwenk, C. (1988). The cognitive perspective on strategic decision making. Journal of Management Studies, 25(1), 41-55.

Schwenk, C. (1995). Strategic decision making. Journal of management, 21(3), 471- 493.

Simon, H. (1947). Administrative behavior. [Las citas y referencias corresponden a la versión en castellano: "El Comportamiento administrativo", 1979]. Barcelona. Biblioteca de economía de la Empresa-Aguilar.

Simon, M. \& Houghton, S. (1999). The effects of cognitive heuristics on strategic action: Overcoming the risk hurdle to first move. New England Journal of Entrepreneurship, 2(1), 34-44.

Simon, M., Houghton, S., \& Aquino, K. (2000). Cognitive biases, risk perception, and venture formation: How individuals decide to start companies. Journal of Business Venturing, 15(2), 113-134.

Tversky, A. \& Kahneman, D. (1973). Availability: A heuristic for judging frequency and probability. Cognitive Psychology, 5(2), 207-232. 
Tversky, A. \& Kahneman, D. (1991). Loss aversion in riskless choice: A reference-dependent model. The quarterly journal of economics, 106(4), 1039-1061.

Urra, J., Medina, A., \& Acosta, A. (2011). Heurísticos y sesgos cognitivos en la dirección de empresas: un meta-análisis. Revista venezolana de gerencia, 16(55), 390-419.

Useche, A. (2014). Exceso de confianza y optimismo en las decisiones de presupuesto de capital: las finanzas corporativas desde un enfoque centrado en el comportamiento. Universidad \& Empresa, 16(26), 95-116.

Vidar, S. \& Lechne, Ch. (2013). Cognitive biases, organization, and entrepreneurial firm survival. European Management Journal, 31(3), 278-294.

Weick, K. (1969) The social psychology of organizing. [Las citas y referencias corresponden a la versión en castellano: "Psicología social del proceso de organización", 1982]. México. Fondo Educativo Interamericano, S. A.

Wilson, T., Centerbar, D., \& Gilbert, D. (2005). The pleasures of uncertainty: Prolonging positive moods in ways people do not anticipate. Journal of Personality and Social Psychology, 88(1), 5-21.

Zapata, G. \& Canet, M. (2009). La cognición del individuo: reflexiones sobre sus procesos e influencia en la organización, Espacio Abierto, 18(2), 235-256.

Zapata, G. \& Hernández, A. (2014) La empresa: diseño, estructuras y formas organizativas. Segunda edición. Barquisimeto. Fondo Editorial UCLA.

Zapata, G, Mirabal, A. \& Canet, M. (2015) El entorno de la organización: un estudio de sus tipologías y su vinculación con la percepción directiva y el diseño organizativo. Ciencia y Sociedad, 40(4), 785-822.

\section{Datos de filiación}

Lorena Barón. Magíster Scientiarum en Gerencia Financiera por la Universidad Centroccidental Lisandro Alvarado; Profesora-investigadora titular del Decanato de Ciencias Económicas y Empresariales de la Universidad Centroccidental Lisandro Alvarado, Venezuela. E-mail: lorenabaron@gmail.com.

Dr. Gerardo Zapata Rotundo. Analista de Sistemas (UCLA, 1985). Licenciado en Contaduría Pública (UCLA, 1993). Especialista en Auditoría (UCLA, 1998); Doctor en Dirección de Empresas (Universidad de Valencia, España, 2006); Docente Titular del Decanato de Ciencias Económicas y Empresariales de la Universidad Centroccidental Lisandro Alvarado (UCLA); Coordinador del Area de Gerencia, Estrategia y Cooperación del Programa del Doctorado en Ciencias Administrativas y Gerenciales de la UCLA/DCEE. Premio anual de investigación "Lisandro Alvarado", Ediciones 2015 y 2016. Se desempeña en la línea de investigación de Organización y Diseńo de la Empresa y Teorías de las Organizaciones; con publicaciones en revistas científicas nacionales e internacionales y presentación de ponencias en diferentes congresos. Email: zapager@yahoo.com 